\title{
Coumarin Communication Along the Microbiome-Root-Shoot Axis
}

\author{
Max J.J. Stassen, ${ }^{1}$ Shu-Hua Hsu, ${ }^{1,2}$ Corné M.J. Pieterse ${ }^{(1)},{ }^{1}$ and loannis A. Stringlis (1D ${ }^{1, *}$
}

Plants shape their rhizosphere microbiome by secreting root exudates into the soil environment. Recently, root-exuded coumarins were identified as novel players in plant-microbiome communication. Beneficial members of the rootassociated microbiome stimulate coumarin biosynthesis in roots and their excretion into the rhizosphere. The iron-mobilizing activity of coumarins facilitates iron uptake from the soil environment, while their selective antimicrobial activity shapes the root microbiome, resulting in promotion of plant growth and health. Evidence is accumulating that, in analogy to strigolactones and flavonoids, coumarins may act in microbiome-to-root-to-shoot signaling events. Here, we review this multifaceted role of coumarins in bidirectional chemical communication along the microbiome-root-shoot axis.

\section{The Microbiome-Root-Shoot Axis}

Nutrients are essential for all organisms to survive, grow, and reproduce. Multicellular eukaryotes have evolved dedicated organs to facilitate nutrient uptake, such as the animal gut and the plant root system. These organs closely collaborate with specialized microbiota (see Glossary) to enhance mobilization and uptake of often scarcely available nutrients. Advances in genomics technologies allowed the in-depth analysis of host-associated microbiomes, revolutionizing the fields of plant and animal biology [1-3]. The microbiome significantly expands the genomic potential of its eukaryotic host, because it encodes functions that support host nutrition, growth, development, and immunity. Microbiome functions represented by microbiota on plant roots and in the animal gut share striking similarities [1,4]. Hence, the root system has been referred to as 'the inverted gut' [5].

In animal and human microbiome biology, the microbiome-gut-brain axis represents the bidirectional biochemical communication between gut microbiota and the central nervous system in the brain, influencing processes ranging from food digestion to mental health (Figure 1, Key Figure) $[6,7]$. Gut microbiota influences the brain not only through the production and alteration of neurotransmitters, such as serotonin and acetylcholine, and short-chain fatty acids, but also through modulation of the immune status of the mucosal membrane, as inflammation of this digestive tract lining can dysregulate the enteric nervous system $[6,7]$. Recently, it was shown in mice that microbiome-derived carnitine-like molecules can directly influence brain functions [8]. In turn, the brain can influence the gut-residing microbiota via neurotransmitters that are perceived by microbial receptors, as found in Pseudomonas spp. and Escherichia coli for the animal neurotransmitters GABA and (nor)adrenaline, respectively [6,7]. Additionally, the brain can modulate gut microbiota through altered gut motility and secretion of acids, mucus, and bicarbonates, therewith exposing gut microbiota to selective environments [6,7].

The root-associated microbiota bears functions reminiscent of those exerted along the human microbiome-gut-brain axis. In plant microbiome biology, bidirectional biochemical communication also shapes root microbiome composition to enhance life-supporting microbiome functions

\section{Highlights}

Bidirectional signaling occurs along the microbiome-root-shoot axis in plants much akin to that along the human microbiome-gut-brain axis.

Production of plant secondary metabolites elicited by rhizosphere microbiota, such as coumarins, can directly impact the composition and activity of the microbial community.

Microbially elicited production of secondary metabolites in the roots can act as intertissue messengers in plants.

Coumarins are the 'new kids on the block' in the chemical communication along the microbiome-root-shoot axis.

${ }^{1}$ Plant-Microbe Interactions, Department of Biology, Science for Life, Utrecht University, Padualaan 8, 3584, CH, Utrecht, The Netherlands ${ }^{2}$ Department of Agronomy, National Taiwan University, No. 1, Section 4, Roosevelt Road, 10617, Taipei, Taiwan

${ }^{*}$ Correspondence:

I.Stringlis@uu.nl (I.A. Stringlis). 


\section{Key Figure}

\section{The Human Microbiome-Gut-Brain Axis versus the Plant Microbiome- Root-Shoot Axis}

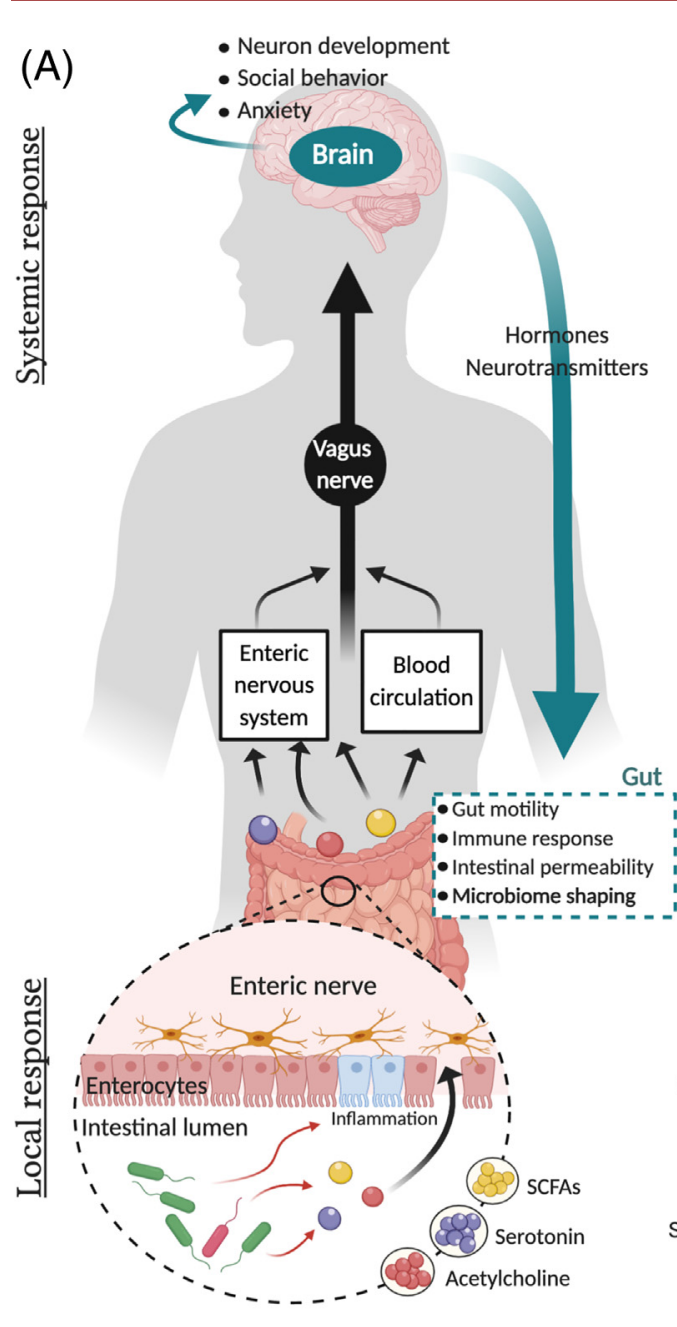

\section{Glossary}

Common symbiosis pathway: a molecular signaling pathway that is essential for plants to associate with AM fungi and for legumes specifically to engage in symbiosis with nitrogen-fixing rhizobacteria. This signaling pathway contains a set of conserved genes that are shared by the rhizobial and mycorrhizal associations. Upon perception of a microbial signal at the plasma membrane, a series of signal transduction cascades is activated involving the production of calcium oscillations, which are perceived by $\mathrm{Ca}^{+} /$ calmodulin-dependent protein kinase (CCaMK). After this, there is transcriptional regulation of transcription factors and genes that coordinate the formation of symbiosis.

Defense priming: a physiological state of the plant following root colonization by selected beneficial microbes or treatment with natural/synthetic compounds characterized by the launching of a faster and stronger defense response, resulting in enhanced resistance against future pathogen or insect attack or enhanced tolerance to abiotic stresses.

Microbiome: the totality of microbial genomes and the functional traits of the microbiota present in a specific host tissue.

Microbiome-gut-brain axis: the bidirectional communication between the gastrointestinal microbiota, the gut, and the central nervous system (brain) involving signals from the neuron system, immune system, and endocrine system that affect gut behavior, and the emotional status and fitness of the host. Microbiome-root-shoot axis: concept describing the bidirectional communication between rhizosphere microbiota, the plant root, and the aerial shoot tissue involving plant- or microbederived signals that affect plant growth, nutrition, and health.

Microbiota: the combined population of all commensal, symbiotic, and pathogenic microbial organisms (including archaea, bacteria, fungi, and protists) that live in or on a host, such as the rhizosphere microbiota or gut microbiota.

\section{Phenylpropanoid pathway: a} secondary metabolite biosynthesis pathway in plants that generates a range of aromatic metabolites. Products of this pathway have roles in the resistance of plants to (a)biotic stresses, and in plant 
for the host plant (Figure 1). For instance, following foliar pathogen infection, plants recruit beneficial microbes to their root system, which in turn can enhance plant immunity $[9,10]$. Recent studies highlighted the link between plant stress and the assembly of beneficial root microbiomes that aid in the alleviation of the stress at hand [11-14]. Often, stress-induced changes in root exudation of carbon (C) sources, signaling molecules, or selective secondary metabolites are important mediators in this process $[15,16]$. In this review, we highlight how selected microbially induced plant signaling compounds, such as strigolactones, flavonoids, and coumarins, act as mediators in the communication between roots and shoots along the microbiome-rootshoot axis. In this context, special emphasis is placed on coumarins, which recently emerged as important players in plant-microbiome interactions.

\section{Plant Exudates}

As the 'inverted gut' of plants, roots can influence the rhizosphere to achieve optimal conditions for nutrient uptake and defense. This is vital, since bioavailability of essential nutrients, such as inorganic phosphate $(\mathrm{Pi})$, nitrogen $(\mathrm{N})$, and iron $(\mathrm{Fe})$, is often limited $[17,18]$. Plants release chemical compounds via their roots that aid in: (i) acquiring essential mineral nutrients from recalcitrant pools in the soil; (ii) establishing mutualistic plant-microbe interactions; and (iii) inhibiting deleterious pathogens in the rhizosphere $[9,16,18-20]$. The nutrient status of the plant has a profound effect on the exudation pattern of roots [19,21] and, consequently, influences plant-microbe relations in the rhizosphere $[13,17,19,22,23]$. During nutrient stresses, plants secrete increased amounts of specific secondary metabolites into the rhizosphere (e.g., strigolactones under Pi stress, flavonoids under $\mathrm{N}$ stress, and coumarins and selected flavonoids under Fe stress) [24-27]. Besides their functions in the mobilization or uptake of the respective scarce mineral nutrients, these secondary metabolites have profound effects on plant-microbiome interactions in the rhizosphere, either through signaling or selective antimicrobial activities [21,28-31].

\section{Strigolactones}

During Pi or N scarcity and under salt or drought stress, plants increase their strigolactone production, often via activation of strigolactone biosynthesis genes in the roots [26,32,33]. Strigolactones are synthesized from carotenoid precursors that, through multiple oxidative cleavings by the carotenoid cleavage dioxygenases CCD7 and CCD8, result in carlactone. Subsequent catalysis of carlactone by cytochrome P450 monooxygenases, such as MAX1, yields different strigolactones (Figure 2) [26]. Their relevance in salt and drought stress is exemplified by Arabidopsis thaliana (hereafter arabidopsis) mutants of the strigolactone responsive factor MAX2. These max2 mutants showed higher sensitivity to drought and salinity stress, caused by slower stomatal closure and increased water loss during dehydration, compared with wild-type plants [34]. Increased strigolactone secretion during Pi limitation represents a strategy of stressed plants to actively recruit arbuscular mycorrhizal (AM) fungi that aid in plant Pi uptake (Figure 3) $[32,35]$. The effect of AM symbiosis on the Pi nutrient status of the plant relies on the activity of fungal phosphate transporters in the mycorrhizal network, which in turn is influenced by host genotype, as found in maize [36]. Strigolactones act as host detection cues for AM fungi and, therefore, are important signals in the presymbiotic phase of the host-mycorrhiza interaction [29,32,37].

propagate systemic signals that modify root structure and cause changes in the rhizosphere environment through changes in root exudation, resulting in enhanced nutrient uptake and shaping of the root-associated microbiome. Black arrows indicate movement of microbe-derived signals, blue arrows indicate brain/shoot-derived signals, and red arrows indicate stimulations. Broken arrows indicate possible interactions, while solid arrows indicate known interactions. Abbreviations: BGLU42, $\beta$-glucosidase BGLU42; CLEs, CLAVATA3/ESR-related peptides; CSP, common symbiosis pathway; F6'H1, feruloyl-CoA 6'-Hydroxylase1. Figure was designed using Biorender (https://biorender.com). structural support and stress adaptation. Phenylpropanoid compounds are derived from the amino acid phenylalanine, the end-product of the shikimate pathway, through deamination by phenylalanine ammonia lyase (PAL). Phenylpropanoid natural compounds include hydroxycinnamic acid, flavonoid/isoflavonoids, lignans/ lignin, coumarins, and stilbenes. 


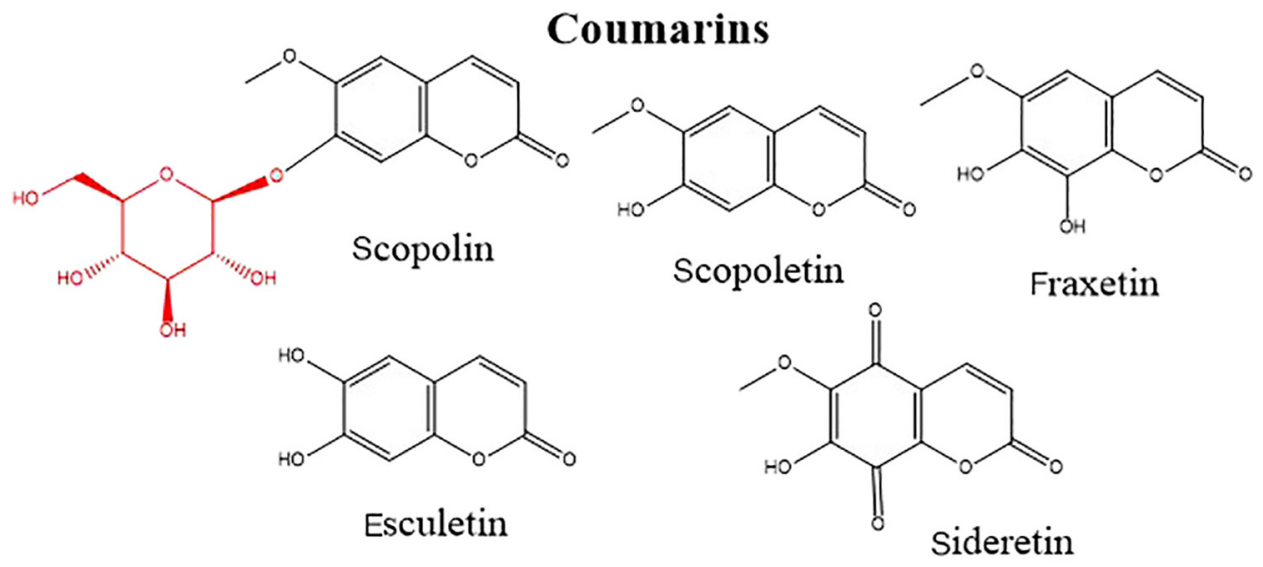

\section{Flavonoids}<smiles>O=c1c(O)c(-c2ccc(O)c(O)c2)oc2cc(O)cc(O)c12</smiles>

\section{Strigolactones}

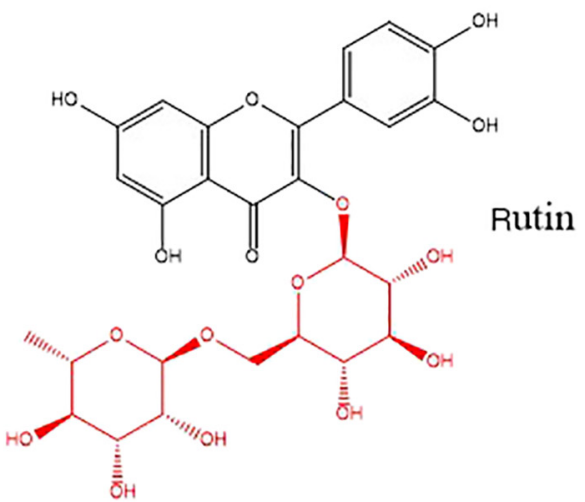<smiles></smiles><smiles>[R12][Z10]#CC1C(=O)O[C@H]2c3ccccc3C[C@H]12</smiles>

Figure 2. Coumarins, Flavonoids, and Strigolactones. Structure formulas of the coumarins scopolin (a coumarin glycoside), scopoletin, fraxetin, esculetin, and sideretin; the flavonoids quercetin and rutin (a quercetin glycoside); the natural strigolactone strigol; and the synthetic strigolactone GR24. Sugar moieties in glycosides are highlighted in red. All structures were created in ChemDraw Professional version 17.

Upon perception, strigolactones induce extensive hyphal branching in the AM fungus, which increases the frequency of host contact and infection [38,39]. Vice versa, AM fungi upregulate strigolactone biosynthesis gene expression in the host root, therewith promoting symbiosis [33]. For instance, maize plants with impaired strigolactone biosynthesis displayed less colonization sites of AM Gigaspora rosea compared with wild-type plants [39], highlighting the role of strigolactones in the establishment of host-mycorrhiza interactions. Interestingly, in later stages of the symbiosis, strigolactone biosynthesis appears to be downregulated $[24,40]$. This effect can sometimes be seen systemically, and is thought to be part of a negative autoregulation of the mycorrhization process [24]. Non-host plants, such as arabidopsis, can also upregulate strigolactone biosynthesis gene expression in response to AM fungi perception [41]. However, 


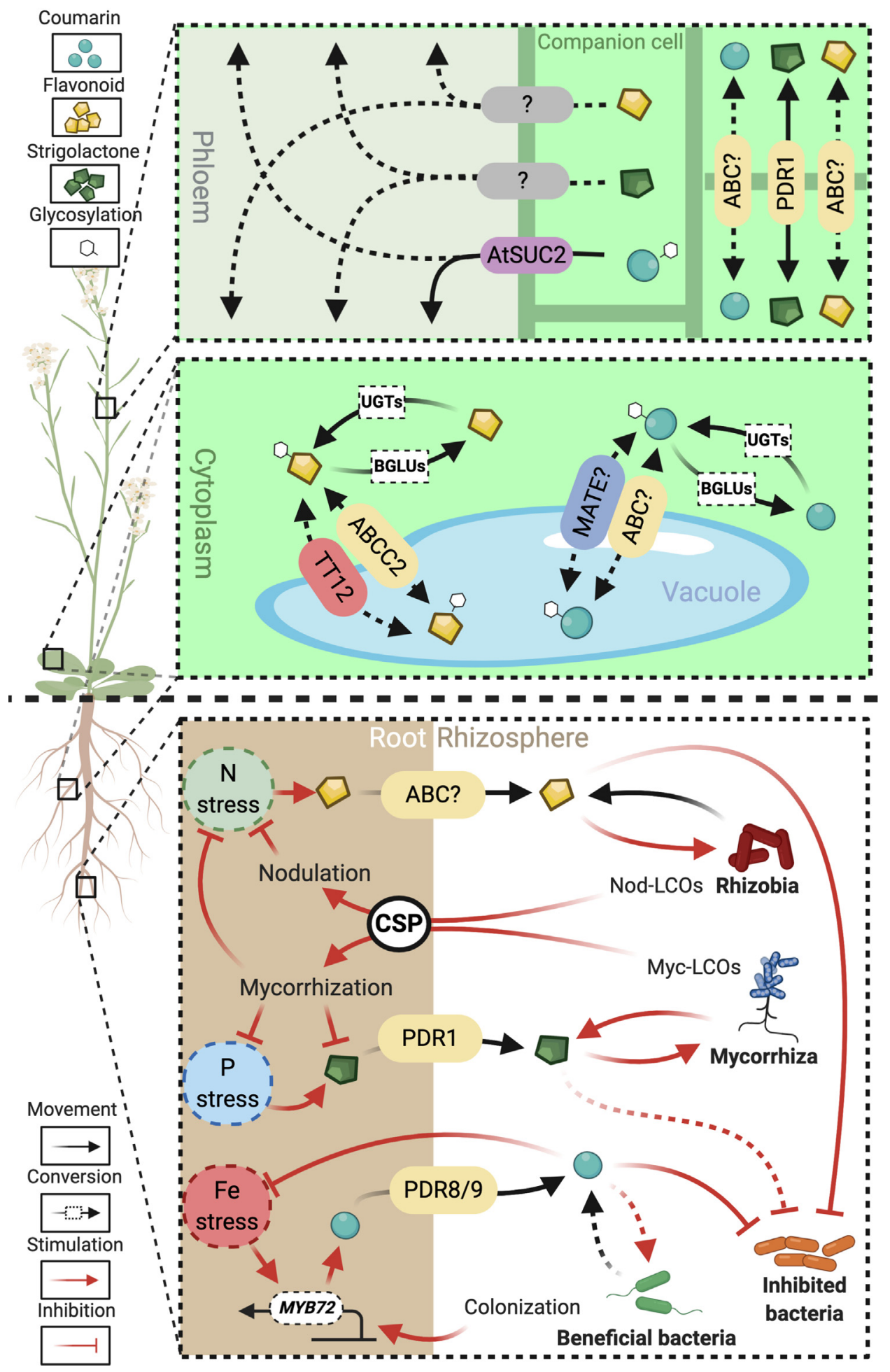

Trends in Plant Science

(See figure legend at the bottom of the next page.) 
such non-host plant-microbe interactions are often incompatible and can even negatively impact plant performance $[41,42]$.

Flavonoids

Flavonoids are secondary metabolites with antioxidant properties that are associated with a range of adaptive responses to abiotic stresses, including UV radiation, and $\mathrm{Pi}$ and $\mathrm{N}$ deficiencies. Flavonoids also have an important role in the chemical communication between plant roots and symbionts, such as nitrogen-fixing Rhizobium bacteria (Figure 3) [28,43,44]. Flavonoids are derived from the phenylpropanoid pathway, a comprehensive supply chain of secondary metabolites responsive to (a)biotic stresses (Figure 4) [45]. Flavonoid production starts with CHALCONE SYNTHASE (CHS), which converts $p$-coumaroyl-CoA, the product of the general phenylpropanoid pathway, to naringenin chalcone $[28,45]$. From there, flavonoid biosynthesis branches out into a diverse group of bioactive compounds, including quercetin and rutin (Figure 2).

Flavonoids mediate the initiation of root nodule-forming symbioses between legumes and Rhizobium bacteria, and between actinorhizal plants and Frankia bacteria. In these interactions, the bacterial partners fix atmospheric $\mathrm{N}$ and make it available to the plant as nitrogenous compounds in return for $\mathrm{C}$ sources [28]. In both legume-Rhizobium and actinorhizal plant-Frankia symbioses, plantderived flavonoids act as chemoattractants for the symbionts [46,47]. In Rhizobium, flavonoids induce the production of Nod factors. Upon recognition by the plant, these lipo-chitooligosaccharide signaling molecules activate the symbiotic molecular dialogue between both partners [48]. In case of the symbioses with AM fungi, Frankia and Rhizobium, the symbiotic dialogue is achieved via the common symbiosis pathway, which is conserved among different plant lineages [49]. Recently, a more general role for this pathway has been proposed, as evidenced by a study where colonization of Lotus japonicus by an endophytic Fusarium species was dependent on some of its components [50].

In mycorrhizal symbioses, flavonoids stimulate mycorrhization and induce hyphal branching and spore germination [44]. Flavonoids have also been implicated in plant defense [51]. In arabidopsis, Sclerotinia sclerotiorum pathogenicity is partly dependent on a quercetin dioxygenase gene, found in multiple bacteria and fungi, which degrades quercetin. This weakens the flavonoid defenses of the plant, allowing for successful pathogen infection [52]. In the symbiosis of alfalfa (Medicago

Figure 3. Signaling Compound Transport, Storage, and Rhizosphere Dynamics. From top to bottom: Transport, compartmentalization, and rhizosphere interactions of coumarins, flavonoids, and strigolactones. All compounds are known to be systemically transported in plants, but only for some glycosylated coumarins was it shown that AtSUC2 loads them into the phloem. Strigolactone secretion and short distance transport occurs through the PaPDR1 ATP binding cassette transporter (ABC) transporter. Flavonoid short-distance transport is also hypothesized to occur through ABC transporters. Coumarins are secreted by the pleiotropic drug resistance protein (PDR)8/9 ABC transporters, which might also facilitate their short-distance transport. Coumarins are stored in the vacuole by unknown transporters. Coumarin (de)glycosylation occurs through UDP-dependent glycosyl transferases (UGTs) and $\beta$-glucosidases (BGLUs). Flavonoids also accumulate in the vacuole, a process mediated by transporters transparent testa 12 (TT12) and ABCC2. Under specific nutrient stresses, coumarins, flavonoids, and strigolactones are increasingly produced and secreted into the rhizosphere, where they signal to members of the root microbiota that can aid in alleviating the nutrient stress. Both flavonoids and strigolactones attract beneficial microbes, which then activate the common symbiosis pathway, leading to alleviation of nutrient stress through nodulation or mycorrhization. Coumarins aid in the alleviation of iron (Fe) stress and might also be part of the molecular dialogue between plants and beneficial microbes, since their production is stimulated in response to colonization of the roots by selected beneficial microbiota members. Conversely, coumarins and flavonoids can also directly inhibit deleterious microorganisms, suggesting that these root-secreted secondary metabolites have a role in shaping the root microbiome with benefits for plant health. Broken arrows indicate possible interactions, while solid arrows indicate known interactions. Abbreviations: ABCG, ATP binding cassette transporter G subfamily; CSP, common symbiosis pathway; MATE, multidrug and toxic compound extrusion protein; MYB72, MYB72 transcription factor; Myc-LCO, Myc-lipo-chito-oligosaccharides; Nod-LCO, Nod-lipo-chito-oligosaccharides. Figure was designed with Biorender (https://biorender.com). 


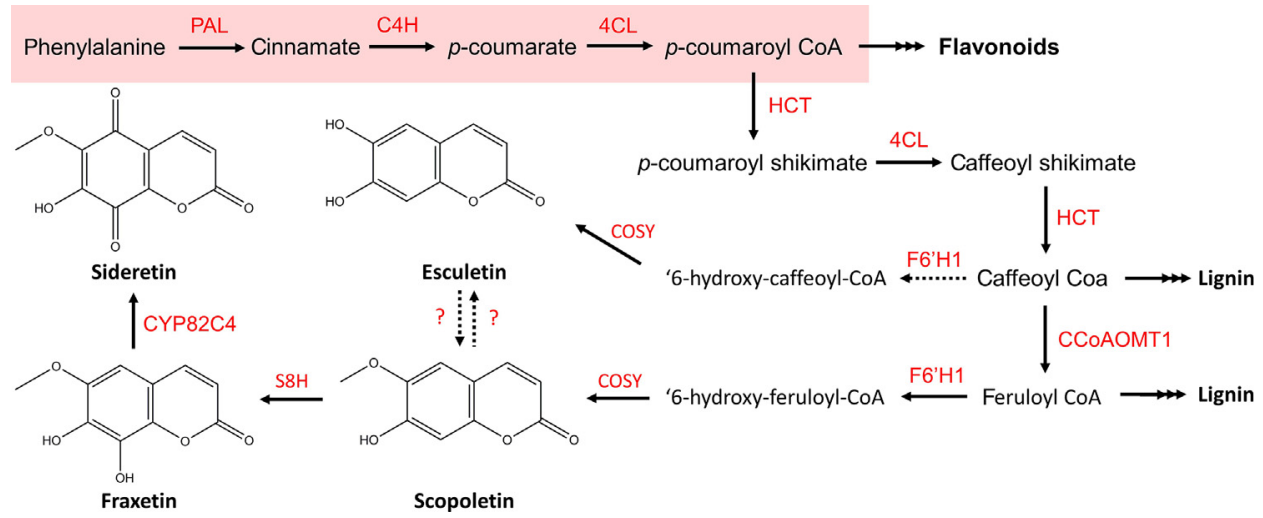

Trends in Plant Science

Figure 4. Coumarin and Flavonoid Biosynthesis. Biosynthesis pathway of coumarins in Arabidopsis. Arrows with names indicate enzymatic activity, broken lines indicate hypothesized conversions. The part of the pathway colored red is the general phenylpropanoid pathway. Flavonoid and lignin biosynthesis branch off from the pathway at $p$-coumaroyl CoA and at caffeoyl CoA, and feruloyl CoA, respectively. Abbreviations: $4 \mathrm{CL}, 4$-coumarate:CoA ligase; $C 3^{\prime} \mathrm{H}, \mathrm{p}$-coumaroyl shikimate 3' hydroxylase; $\mathrm{C} 4 \mathrm{H}$, cinnamate 4-hydroxylase; CCOAOMT, caffeoyl CoA 3-O-methyltransferase; COSY, coumarin synthase; CYP82C4, cytochrome P450 family B2 subfamily C polypeptide 4 enzyme; F6'H1, feruloyl-CoA 6'-Hydroxylase1; HCT, hydroxycinnamol-coenzyme A shikimate/quinate hydroxycinnamoyl-transferase; PAL, phenylalanine ammonia-lyase; S8H, scopoletin 8-hydroxylase. Structures were created in ChemDraw Professional version 17.

sativa) and Rhizobium meliloti, the rhizobium induces the exudation of the flavonoid phytoalexin medicarpin by the plant [53]. Interestingly, $R$. meliloti is resistant to this compound but other rhizobia are not, suggesting a role of this compound in a selective process for suitable symbiotic partners [54]. The nutrient-dependent exudation of flavonoids, their antimicrobial properties, and their plant-microbe signaling functions imply a more profound selective effect on the root microbiome than only symbiosis signaling. However, information on the influence of flavonoids in plant-whole-microbiome communication is limited.

\section{Coumarins}

Plant-derived coumarins have long been known for their antimicrobial activity in plant-pathogen interactions, but their role in nutrient uptake and root-microbiome communication was unearthed only recently [21,55-57]. Due to their multifaceted roles in the interaction of plants with their (a)biotic environment, coumarins emerged as 'new kids on the block' next to the well-established strigolactones and flavonoids. Therefore, here, we discuss the role of coumarins in communication along the microbiome-root-shoot axis and highlight, when possible, analogies with similar roles of strigolactones and flavonoids.

\section{Coumarins: Biosynthesis, Storage, and Secretion}

Coumarins are low-molecular-weight, in some cases fluorescent, plant secondary metabolites that, similar to flavonoids, are synthesized via the phenylpropanoid pathway (Figure 4) [45]. Since coumarins are the focus of this review, we present here their key biosynthetic steps in more detail. Coumarin biosynthesis starts from the general phenylpropanoid pathway, which effectuates the conversion of phenylalanine, the product of the shikimate pathway, to $p$-coumaroyl-CoA (Figure 4). In arabidopsis, the production steps of coumarins from $p$-coumaroyl-CoA until feruloyl CoA are shared with those of lignin. The enzyme FERULOYL-COA 6'-HYDROXYLASE1 (F6'H1), on which the production of coumarins is dependent, converts feruloyl CoA to 6'hydroxy-feruloyl CoA, which then undergoes isomerization of the side-chain and lactonization, resulting in the coumarin scopoletin [58]. This latter step occurs spontaneously via light, but in darkness is mediated by the recently discovered COUMARIN SYNTHASE (COSY) [59]. Then, scopoletin can be hydroxylated by SCOPOLETIN 
8-HYDROXYLASE (S8H), which yields the coumarin fraxetin [60,61]. Finally, coumarin sideretin is produced via oxidation of fraxetin by the cytochrome P450 enzyme CYP82C4 [62]. The COSY enzyme catalyzes the conversion of 6'-hydroxy-caffeoyl-CoA to the coumarin esculetin [59].

In plants, coumarins occur in glycosylated (glycoside) or deglycosylated (aglycone) forms (Figure 2). Glycosylation provides the plant with an easy way to modify molecules. This can be used to limit exposure to certain aglycones, for example, to protect themselves from reactive defense compounds, or to adjust the signaling capabilities of certain compounds. Additionally, compounds in their glycoside form are easier to store and transport [63]. The glycosylation of coumarins occurs in the cytoplasm by UDP-glycosyltransferases [63]. After glycosylation, coumarins are transported to the vacuole, as evidenced for scopolin and esculin in barley and tobacco cells (Figure 3) [64,65]. However, the transporters involved are unknown. ATP-binding cassette (ABC)-transporters and multidrug and toxic compound extrusion proteins are likely candidates, since flavonoids, which occur in similar forms (hydroxylated, methylated, and glycosylated) as coumarins, utilize these types of transporter for vacuolar transport (Figure 3). Upon stress, vacuole-stored coumarin glycosides can be deglycosylated to biologically active aglycone forms that are then released into the rhizosphere $[56,66,67]$. This deglycosylation occurs through $\beta$-glucosidase enzymes (BGLUs). Before a stress, glycosides (in vacuoles) and BGLUs (in cell walls, apoplasts, and endoplasmic reticulum bodies) are spatially separated, but, upon tissue damage due to a stress, they come in contact and deglycosylation occurs [63]. Previously, arabidopsis BGLU21, BGLU22, and BGLU23 were found to hydrolyze scopolin and esculin to scopoletin and esculetin, respectively, in vitro [68]. In arabidopsis roots, Fe-deficiency-induced BGLU42 was demonstrated to convert scopolin to scopoletin, a process that appeared to be required for the secretion of scopoletin from the root into the rhizosphere (Figure 5) [56].

\section{Iron and Coumarins}

Plant survival relies on the successful acquisition of nutrients from their environment and this is facilitated by their 'inverted gut'. Due to its function as a redox catalyst in many cellular processes, $\mathrm{Fe}$ is an essential nutrient for all life on Earth. Although abundantly present in the soil, its bioavailability is often limited because $\mathrm{Fe}$ is poorly soluble at neutral and high $\mathrm{pH}$. As a result, plants evolved ingenious strategies to mobilize and take up poorly available Fe from the soil. Nongrass plants and grass plants evolved different Fe deficiency responses, called Strategy I and Strategy II, respectively, both of which are activated in roots upon sensing low Fe availability [69]. Strategy II grass plants secrete Fe-chelating phytosiderophores into the rhizosphere. These phytosiderophores bind ferric $\mathrm{Fe}\left(\mathrm{Fe}^{3+}\right)$ in the soil, after which the complex is taken up by transporters in the root epidermis [70]. In Strategy I plants, such as arabidopsis, $\mathrm{H}^{+}$-ATPase AHA2 acidifies the rhizosphere, which increases the solubility of $\mathrm{Fe}^{3+}$ in the soil [71]. Consecutively, another membrane-bound protein, FERRIC REDUCTION OXIDASE 2 (FRO2), reduces the now free ferric $\mathrm{Fe}^{3+}$ ions to ferrous $\mathrm{Fe}^{2+}$, the form in which it can be taken up by IRONREGULATED TRANSPORTER1 (IRT1) [72,73]. This process is controlled by FER-LIKE IRONDEFICIENCY INDUCED TRANSCRIPTION FACTOR (FIT), a central regulator of the Fe deficiency response in Strategy I plants [74].

In addition to the canonical Fe-deficiency response genes, phenylpropanoid pathway and coumarin biosynthesis and secretion genes are highly responsive to Fe deficiency, and have also been described to be regulated by FIT, in a manner dependent on the downstream MYB72 transcription factor [75-77]. During Fe limitation, Strategy I plant roots increase the secretion of scopoletin, esculetin, fraxetin, and sideretin (Figure 2). Arabidopsis mutants blocked in coumarin biosynthesis or secretion are impaired in their survival in Fe-deprived alkaline soils [60,62,78,79]. Coumarin secretion relies on the activity of the membrane-bound ABC transporters PDR8 


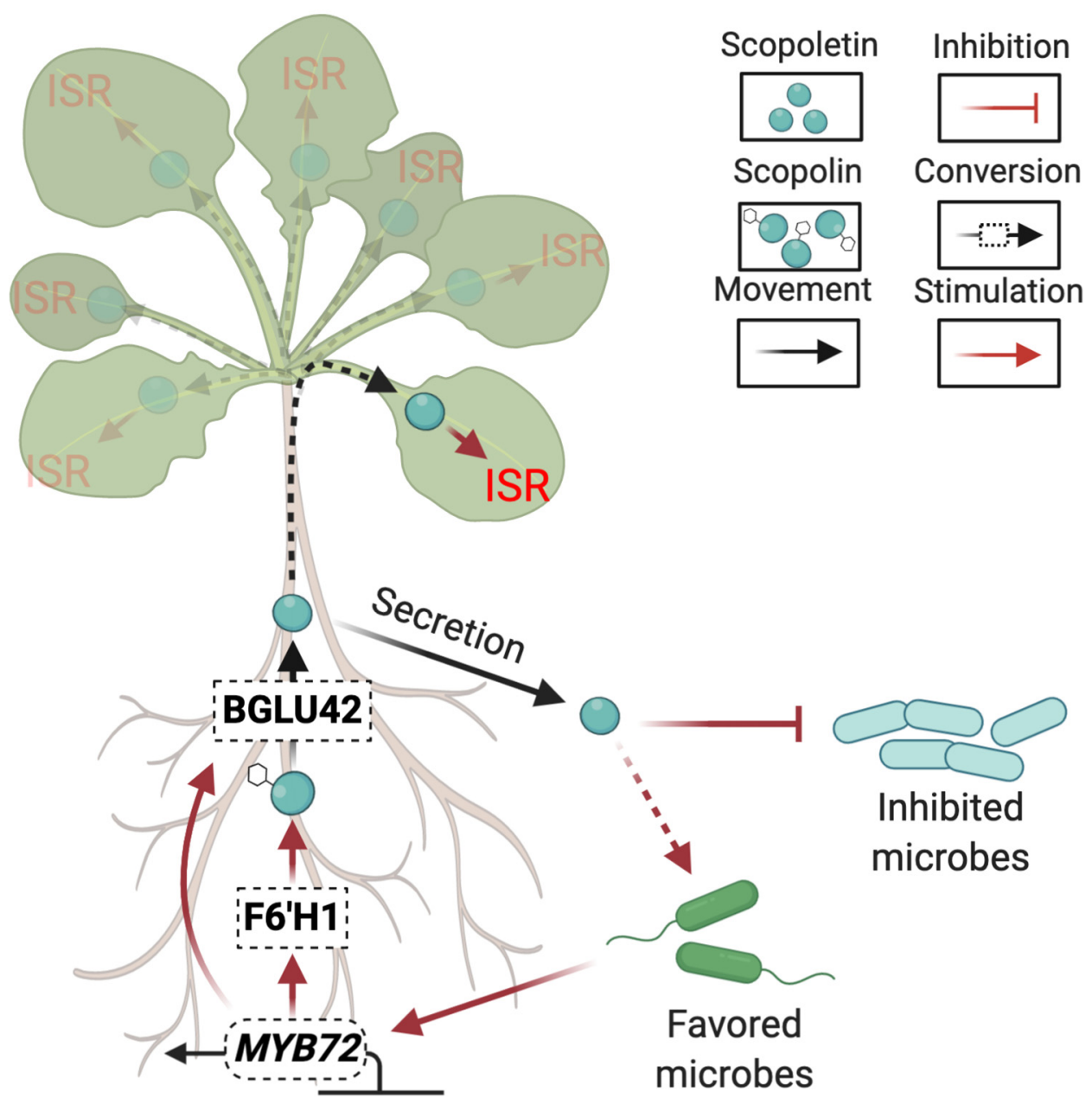

Trends in Plant Science

Figure 5. Coumarins in Bidirectional Signaling Along the Microbiome-Root-Shoot Axis. Colonization of roots by ISR-eliciting, coumarin-tolerant rhizobacteria stimulates the expression of the root-localized transcription factor MYB72. This transcription factor promotes the production and secretion of the coumarin scopoletin by activating the coumarin biosynthesis gene $F 6^{\prime} H 1$ and the $\beta$-glucosidase gene BGLU42, respectively. BGLU42 converts scopolin to its aglycone scopoletin, after which it is secreted into the rhizosphere. Products of BGLU42 activity, such as scopoletin, are hypothesized to also travel from the root to the shoot, where they may act in the onset of ISR. Root-secreted scopoletin (together with other coumarins) can favor or inhibit different microbes, creating a niche for those tolerant to its antimicrobial activity. This selective role in the rhizosphere could stimulate microbially elicited plant-microbe communication with benefits for plant growth and health. Broken arrows indicate possible interactions, while solid arrows indicate known interactions. Abbreviations: BGLU42, $\beta$-glucosidase 42; F6'H1, feruloyl-CoA 6'-Hydroxylase1; ISR, induced systemic resistance. Figure was designed with Biorender (https://biorender.com).

(scopoletin) and PDR9 (esculetin, fraxetin, and sideretin) (Figure 3) [67,78]. In alkaline conditions, buffering compounds bind the $\mathrm{H}^{+}$secreted by AHA2, impairing their function in solubilizing $\mathrm{Fe}^{3+}$ [71]. The way in which coumarins relieve Fe malnutrition in alkaline conditions requires $\mathrm{FRO} 2$ and IRT1 but not AHA2 functionality [80]. Since these proteins facilitate the reduction and subsequent uptake of $\mathrm{Fe}$, it appears that coumarins function in the step before reduction, in the solubilization of $\mathrm{Fe}$. By binding $\mathrm{Fe}^{3+}$ through high-affinity chelation, coumarins can retrieve $\mathrm{Fe}^{3+}$ from recalcitrant pools, after which previously insoluble $\mathrm{Fe}^{3+}$ becomes soluble through the solubility constant [21]. Then, the Fe can be provided to $\mathrm{FRO}$, restoring Strategy I functionality. 


\section{Coumarins in Root-Microbiome Communication}

Roots interact with both beneficial and harmful microbes and their ability to discriminate between the good and the bad consequently determines the extent by which a plant host benefits from its root microbiome. Biofilm formation at the root-microbiome interface is an early determinant in successful plant-microbe interactions [81]. When grown in high densities, such as in biofilms, many bacteria regulate their interactions and physiological processes through quorum sensing, a process in which bacterial cells communicate with each other through diffusible molecules [81]. In the medical field, coumarins have been identified as effective antibiofilm and antiquorum-sensing compounds [82]. Scopoletin, esculetin, esculin, and fraxin inhibit biofilm formation by several human bacterial pathogens through interference with quorum sensing signaling [82-84]. Esculetin was found to dock on the quorum-sensing TraR protein [85], inhibiting transduction of the signal that is necessary to activate quorum-sensing genes and, resultantly, biofilm formation [83-85]. Interestingly, esculetin could also inhibit biofilm formation of the widespread plant pathogen Ralstonia solanacearum [86], suggesting that coumarins interfere more broadly in biofilm formation in host-microbe interactions. Evidence for species-specific inhibition of biofilm formation was recently provided for certain flavonoids, adding to the notion that plant-exuded molecules may aid in favoring certain microbes over others in the microbiome associated with the plant [87].

Coumarins are known to function as phytoalexins, which are antimicrobial compounds that contribute to plant disease resistance against microbial pathogens [55]. Scopoletin in particular has been thoroughly studied in the context of plant immunity across multiple plant species, where its accumulation has been directly linked to increased resistance against several phytopathogens $[86,88,89]$. The modes of action by which coumarins contribute to disease resistance range from direct effects on microbial membrane integrity [86], to scavenging of reactive oxygen species (ROS) that are produced in response to pathogen infection and help to prevent tissue damage [89]. Catecholic coumarins are known to situationally act as oxidants, and have been shown to produce microbe-inhibiting ROS molecules, such as $\mathrm{H}_{2} \mathrm{O}_{2}[57,90]$. Several secondary metabolites with inhibitory effects have been implied to help shape the plant microbiome $[56,57,91]$. For example, flavonoid-rich root exudates of Erythrina falcata inhibited denitrifying bacteria more strongly compared with nitrifying bacteria in vitro, therewith selecting a microbial community aiding in the need of the plant for nitrate [91].

Considering their antimicrobial activity and abundant secretion into the rhizosphere, coumarins emerged as potential regulators of root microbiome composition [56,57]. Recently, two studies assessed the effect of coumarin biosynthesis mutants on microbial community composition in soil [56] and on a synthetic community (SynCom) composition in a gnotobiotic system [57]. In the rhizosphere, coumarin biosynthesis-impaired $f 6^{\prime} h 1$ plants assembled a different rhizosphere microbiome compared with Col-0 plants [56]. The most strongly promoted genera in coumarinproducing Col-0 plants were those with the ability to promote plant growth, facilitate plant metal uptake, fixate nitrogen in the soil, or display antimicrobial properties [56]. Additionally, two plant beneficial mutualists, Pseudomonas simiae WCS417 (hereafter WCS417) and Pseudomonas capeferrum WCS358, were shown to be resistant to the most abundantly secreted coumarin scopoletin, whereas the soil-borne plant pathogens Fusarium oxysporum and Verticillium dahliae were detrimentally affected by this coumarin [56]. In the SynCom study with different coumarinimpaired mutants ( $f 6^{\prime} h 1, s 8 h$, and cyp82c4) the observation that coumarins affect the composition of the root-associated microbiome under Fe deficiency was confirmed [57]. Specifically, one Pseudomonas isolate was shown to be more abundant in the coumarin biosynthesis and excretion mutants $f 6^{\prime} h 1$ and $p d r 9$, respectively, attributed to a lack of coumarin-dependent ROS generation in these mutants [57]. In addition to flavonoids and coumarins, strigolactones can also affect the rhizosphere microbiome as demonstrated by studies in rice and soybean 
$[92,93]$. Soybean mutants overexpressing or rice mutants defective in strigolactone biosynthesis or signaling harbored differing rhizosphere microbiomes compared with their respective wild types [92,93].

Another important aspect of host-microbe communication in the rhizosphere is chemotaxis, the process by which microbes move along a concentration gradient of a specific chemical [94]. Exudation of plant-derived semiochemicals coordinates the communication between plants and microbes by activating chemotactic responses in specific mutualists. Examples of such semiochemicals are the flavonoids luteolin and rutin, which attract Rhizobium spp. and Bacillus subtilis, respectively, and strigolactones that stimulate colonization of AM fungi $[95,96]$. Coumarins have also been implicated in this process. Scopoletin was shown to deter germinating $F$. oxysporum spores, suggesting that, besides antimicrobial selection and interference with biofilm formation, coumarins also act via chemotaxis in shaping the root-associated microbiome [56]. However, since these studies mostly focused on a selection of compounds and bacteria, the generality/ specificity of these chemotactic responses needs to be further tested. A wide screening involving multiple bacterial taxa and structurally different compounds would increase our understanding of the chemical communication in the rhizosphere.

\section{Coumarins in Microbiome-Root Communication}

Analogous to how the gut microbiota contributes to nutrient absorption and human health, the plant root microbiome influences plant fitness by facilitating nutrient uptake, promoting plant growth, and stimulating plant immunity (Figures 1 and 3 ) $[1,4,16]$. The latter is a direct indication of microbiome-root communication because it develops in the whole plant body in response to colonization of the roots by selected members of the root microbiome. Plant-beneficial rhizobacteria of the genera Pseudomonas and Bacillus, and fungi of the genus Trichoderma [97] emerged as potent activators of induced systemic resistance (ISR), a conserved plant defense mechanism by which plants develop an enhanced defensive capacity against a broad spectrum of plant pathogens [98]. Colonization of the roots by ISR-inducing microbes typically causes extensive transcriptional and metabolomic changes locally in the roots, but not in the leaves. However, these leaves can mount stronger defenses upon pathogen attack, a phenomenon known as 'defense priming' [76,98-100]. Microbially elicited plant secondary metabolites have been shown to have a role in this phenomenon. For instance, the flavonoid rutin (Figure 2) induces defense priming against a range of different pathogens and concurrently stimulates biofilm formation and chemotaxis in mutualistic $B$. subtilis rhizospheric bacteria [95,101]. ISR and defense priming have been characterized in detail for the interaction between arabidopsis and beneficial Pseudomonas and Trichoderma spp. [98]. A key regulator of ISR initiation is the root-specific transcription factor MYB72. MYB72 gene expression is induced in roots in response to colonization by, or following perception of, so far uncharacterized volatile organic compounds of ISRinducing Pseudomonas or Trichoderma sp. strains, and arabidopsis myb72 mutants are unable to mount ISR upon colonization by these beneficial rhizosphere-inhabiting microbes [97,102-104].

As mentioned earlier, MYB72 also regulates the biosynthesis (F6'H1) and secretion (BGLU42) of coumarins in roots under conditions of Fe stress [76]. Interestingly, ISR-inducing microbes also stimulate the biosynthesis and secretion of coumarins in a MYB72-dependent manner, even under conditions of sufficient Fe availability [56]. As a result, the coumarin-inducing mutualists improve Fe nutrition and growth of the plant [105]. Given that the coumarin-inducing mutualists are themselves tolerant to the antimicrobial effect of the coumarins, this process may be part of a feed-forward loop in which the mutualist gains niche establishment on the root surface, while the plant is rewarded with enhanced Fe nutrition and protection via the elicitation of ISR (Figures 3 and 5) $[56,76,98]$. 


\section{Coumarins in Root-Shoot Communication}

Along the microbiome-root-shoot axis, signals initiated during interactions at the rootmicrobiome interface can have an important role in the systemic communication between roots and shoots (Figure 1). A classic example is autoregulation of nodulation, wherein nodulating roots produce small peptide signals, known as rhizobia-induced CLAVATA3/ESR-related peptides (CLEs), which translocate to the shoot, where they are recognized by a CLAVATA1like receptor complex. This complex subsequently produces a shoot-root inhibitory signal molecule, previously thought to be a cytokinin but recently proposed to be a miRNA, which in turn regulates the number of formed nodules $[106,107]$. Grafting experiments in soybean showed that glycerol-3-phosphate, a mobile signal associated with systemic acquired resistance (SAR), is produced in the shoot upon root colonization by an incompatible rhizobium, and is needed to exclude this bacterium from the nodule, pointing towards reciprocal signaling from root to shoot [108]. A recent study showed that local interaction of tomato roots with a soil-derived microbial community can result in metabolomic and transcriptomic changes in distal root and shoot parts. Another mobile SAR signal, azelaic acid, was implicated as a signaling molecule in this process, traveling from root to shoot in its glycosylated form, before relocation to and exudation in its aglycone form through the roots. These studies exemplify the capabilities of the rhizosphere microbiome to systemically affect distal root and shoot tissue through the induction of mobile signals.

In the interaction between plant roots and ISR-inducing rhizosphere microbes, the mobile signal translating rhizobacteria-induced transcriptional changes in the root to defense priming in shoot has remained elusive. Coumarins emerged as potential candidates, since overexpression of the coumarin-deglycosylating enzyme BGLU42 in arabidopsis conferred constitutive ISR to different types of pathogen [76]. BGLU42 gene expression is activated in arabidopsis roots in response to colonization by ISR-inducing rhizobacteria in a MYB72-dependent manner [70]. The main target of BGLU42 is scopolin, which is converted into its aglycone scopoletin by BGLU42 activity [56]. This BGLU42-mediated deglycosylation results in scopoletin mobility, because it becomes abundantly secreted into the rhizosphere. It is tempting to speculate that coumarins like scopoletin become uploaded in the root vasculature where they may serve as mobile signals that contribute to enhanced defense in aboveground plant tissues (Figure 5).

Evidence for a role of coumarins in root-shoot communication is scarce. In arabidopsis, the sucrose transporter AtSUC2 was implicated in the systemic transport of fraxin and esculin from leaves to the root tips [109]. In the case of flavonoids, long-distance transport has been demonstrated and is hypothesized to occur via ABC transporters [110]. Systemic transport is structure dependent, because the flavonoid quercetin is not a target for this transport, while its precursor dihydroxyquercetin is [110]. Interestingly, short-distance transport and secretion of strigolactones is effectuated by a member of the ABC transporter family (PDR1) [111]. ABC transporters PDR8 and PDR9 have been demonstrated to translocate specific coumarins from the root into the rhizosphere $[67,78,80]$. Hence, a role for these transporters in coumarin root-shoot communication would be worth investigating (Figure 4), either by using mutant plants for transporter proteins, micrografting, or using compounds that inhibit transporter activity to unravel their spatial activity during long-distance transport of microbially induced signaling compounds.

\section{Concluding Remarks}

Advances in next-generation sequencing technologies and novel experimental approaches are starting to gradually decode the 'hidden' communication between plants and their rhizosphereinhabiting microbes. In analogy to the human microbiome-gut-brain axis, the microbiomeroot-shoot axis displays extensive bidirectional signaling with positive effects on host nutrition
Outstanding Questions

What are the molecular mechanisms underlying the interplay between nutrient stress, secondary metabolite secretion, and root microbiome recruitment?

By which mechanisms do plantderived secondary metabolites, such as coumarins, exert their selective pressure on rhizosphere microbiota, and which microbes are typically tolerant or resistant?

How do selectively antimicrobia secondary metabolites function in the molecular dialogue between plant and root-associated mutualists?

Do coumarins function as mobile signals in root-shoot signaling during beneficial microbe-elicited ISR? 
and health. The functions of plant metabolites released in the soil are as diverse as they are numerous. Among them, strigolactones, flavonoids, and coumarins inhibit the growth of pathogens, select and nurture the growth of plant growth-promoting microbes, alter physiological conditions of the soil, and are highly instrumental in the chemical communication at the rootmicrobiome interface. This review focused on the role of coumarins in plant-microbe interactions, moving from a more traditional view of coumarins as antimicrobial phytoalexins, to a more recent insight highlighting their importance in shaping the root-associated microbiome composition, $\mathrm{Fe}$ mobilization and uptake, and the onset of ISR, therewith benefiting plant growth and immunity. Future research efforts (see Outstanding Questions) should aim to elucidate how these rootderived 'neurotransmitters' affect the aboveground plant 'brain' to ultimately exploit the functions of the 'inverted gut' microbiota and achieve optimal plant fitness.

\section{Acknowledgments}

This work was supported by NWO Gravity Programme MiCRop: Harnessing the second genome of plants (Grant 024.004.014; C.M.J.P. and I.A.S), Dutch Technology Foundation TTW, which is part of The Netherlands Organization of Scientific Research (NWO) and partly funded by the Ministry of Economic Affairs (Back2Roots Grant 14219; C.M.J.P), and a scholarship from the Ministry of Science and Technology of Taiwan (108-2917-I-002-003, 2019; S.H).

\section{References}

1. Berendsen, R.L. et al. (2012) The rhizosphere microbiome and plant health. Trends Plant Sci. 17, 478-486

2. Müller, D.B. et al. (2016) The plant microbiota: systems-level insights and perspectives. Annu. Rev. Genet. 50, 211-234

3. Bulgarelli, D. et al. (2013) Structure and functions of the bacterial microbiota of plants. Annu. Rev. Plant Biol. 64, 807-838

4. Hacquard, S. et al. (2015) Microbiota and host nutrition across plant and animal kingdoms. Cell Host Microbe 17, 603-616

5. Geldner, N. (2013) The endodermis. Annu. Rev. Plant Biol. 64, $531-558$

6. Carabotti, M. et al. (2015) The gut-brain axis: interactions between enteric microbiota, central and enteric nervous systems. Ann. Gastroenterol. 28, 203-209

7. Cryan, J.F. et al. (2019) The microbiota-gut-brain axis. Physiol. Rev. 99, 1877-2013

8. Hulme, H. et al. (2020) Microbiome-derived carnitine mimics as previously unknown mediators of gut-brain axis communication. Sci. Adv. 6, eaax6328

9. Berendsen, R.L. et al. (2018) Disease-induced assemblage of a plant-beneficial bacterial consortium. ISME J. 12, 1496-1507

10. Yuan, J. et al. (2018) Root exudates drive the soil-borne legacy of aboveground pathogen infection. Microbiome 6, 1-12

11. Stringlis, I.A. et al. (2018) Root transcriptional dynamics induced by beneficial rhizobacteria and microbial immune elicitors reveal signatures of adaptation to mutualists. Plant J. 93, 166-180

12. Finkel, O.M. et al. (2019) The effects of soil phosphorus content on plant microbiota are driven by the plant phosphate starvation response. PLoS Biol. 17, 1-34

13. Bakker, P.A.H.M. et al. (2018) The soil-borne legacy. Cell 172 , 1178-1180

14. Hiruma, K. et al. (2016) Root endophyte Colletotrichum tofieldiae confers plant fitness benefits that are phosphate status dependent. Cell 165, 464-474

15. Bais, H.P. et al. (2006) The role of root exudates in rhizosphere interactions with plants and other organisms. Annu. Rev. Plant Biol. 57, 233-266

16. Pascale, A. et al. (2020) Modulation of the root microbiome by plant molecules: the basis for targeted disease suppression and plant growth promotion. Front. Plant Sci. 10, 1741

17. Castrillo, G. et al. (2017) Root microbiota drive direct integration of phosphate stress and immunity. Nature 543, 513-518

18. Verbon, E.H. et al. (2017) Iron and immunity. Annu. Rev. Phytopathol. 55, 355-375

19. Sasse, J. et al. (2018) Feed your friends: do plant exudates shape the root microbiome? Trends Plant Sci. 23, 25-41

20. Rolfe, S.A. et al. (2019) Crying out for help with root exudates: adaptive mechanisms by which stressed plants assemble health-promoting soil microbiomes, Curr. Opin. Microbiol. 49 , 73-82

21. Tsai, H.H. et al. (2017) Mobilization of iron by plant-borne coumarins. Trends Plant Sci. 22, 538-548

22. Hu, L. et al. (2018) Root exudate metabolites drive plant-soil feedbacks on growth and defense by shaping the rhizosphere microbiota. Nat. Commun. 9, 1-13

23. Chutia, R. et al. (2019) Iron and phosphate deficiency regulators concertedly control coumarin profiles in Arabidopsis thaliana roots during iron, phosphate, and combined deficiencies. Front. Plant Sci. 10, 1-12

24. García-Garrido, J.M. et al. (2009) Strigolactones, signals for parasitic plants and arbuscular mycorrhizal fungi. Mycorrhiza $19,449-459$

25. Tsai, H.H. and Schmidt, W. (2017) One way. Or another? Iron uptake in plants. New Phytol. 214, 500-505

26. Koltai, H. and Prandi, C. (2019) Strigolactones - Biology and Applications, Springer Nature

27. Coronado, C. et al. (1995) Alfalfa root flavonoid production is nitrogen regulated. Plant Physiol. 108, 533-542

28. Abdel-Lateif, K. et al. (2012) The role of flavonoids in the establishment of plant roots endosymbioses with arbuscular mycorrhiza fungi, rhizobia and Frankia bacteria. Plant Signal. Behav. 7, 636-641

29. De Cuyper, C. and Goormachtig, S. (2017) Strigolactones in the rhizosphere: friend or foe? Mol. Plant-Microbe Interact. 30, 683-690

30. Marzec, M. (2016) Strigolactones as part of the plant defence system. Trends Plant Sci. 21, 900-903

31. Ta, C.A.K. and Arnason, J.T. (2016) Mini review of phytochemicals and plant taxa with activity as microbial biofilm and quorum sensing inhibitors. Molecules 21, 1-26

32. Al-Babili, S. and Bouwmeester, H.J. (2015) Strigolactones, a novel carotenoid-derived plant hormone. Annu. Rev. Plant Biol. 66, 161-186

33. López-Ráez, J.A. et al. (2015) Differential spatio-temporal expression of carotenoid cleavage dioxygenases regulates apocarotenoid fluxes during AM symbiosis. Plant Sci 230, 59-69

34. Van Ha, C. et al. (2014) Positive regulatory role of strigolactone in plant responses to drought and salt stress. Proc. Natl. Acad. Sci. U. S. A. 111, 851-856

35. Yoneyama, K. et al. (2007) Phosphorus deficiency in red clove promotes exudation of orobanchol, the signal for mycorrhizal symbionts and germination stimulant for root parasites. Planta 225, 1031-1038

36. Sawers, R.J.H. et al. (2017) Phosphorus acquisition efficiency in arbuscular mycorrhizal maize is correlated with the abundance 
of root-external hyphae and the accumulation of transcripts encoding PHT1 phosphate transporters. New Phytol. 214, 632-643

37. López-Ráez, J.A. et al. (2017) Strigolactones in plant interactions with beneficial and detrimental organisms: the Yin and Yang. Trends Plant Sci. 22, 527-537

38. Mori, N. et al. (2016) Carlactone-type strigolactones and their synthetic analogues as inducers of hyphal branching in arbuscular mycorrhizal fungi. Phytochemistry 130, 90-98

39. Gomex-Roldan, V. et al. (2007) Strigolactones: promising plant signals. Plant Signal. Behav. 2, 163-164

40. Lendzemo, V. et al. (2009) Striga seed-germination activity of root exudates and compounds present in stems of Atriga host and nonhost (trap crop) plants is reduced due to root colonization by arbuscular mycorrhizal fungi. Mycorrhiza 19, 287-294

41. Fernández I. etal. (2019) Molecular dialogue between arbuscular mycorrhizal fungi and the nonhost plant Arabidopsis thaliana switches from initial detection to antagonism. New Phytol. 223, 867-881

42. Cosme, M. et al. (2018) Non-mycorrhizal plants: the exceptions that prove the rule. Trends Plant Sci. 23, 577-587

43. Mollavali, M. et al. (2018) Nitrogen form and mycorrhizal inoculation amount and timing affect flavonol biosynthesis in onion (Allium cepa L.). Mycorrhiza 28, 59-70

44. Cesco, S. et al. (2010) Release of plant-borne flavonoids into the rhizosphere and their role in plant nutrition. Plant Soil 329 $1-25$

45. Vogt, T. (2010) Phenylpropanoid biosynthesis. Mol. Plant 3 , 2-20

46. Dharmatilake, A.J. and Bauer, W.D. (1992) Chemotaxis of Rhizobium meliloti towards alfalfa roots. Appl. Environ. Microbiol. 58 1153-118

47. Popovici, J. et al. (2010) Differential effects of rare specific flavonoids on compatible and incompatible strains in the Myrica gale-Frankia actinorhizal symbiosis. Appl. Environ. Microbiol. 76, 2451-2460

48. Fliegmann, J. and Bono, J.J. (2015) Lipo-chitooligosaccharidic nodulation factors and their perception by plant receptors. Glycoconj. J. 32, 455-464

49. Radhakrishnan, G.V. et al. (2020) An ancestral signalling pathway is conserved in plant lineages forming intracellular symbioses. Nat. Plants 6, 280-289

50. Skiada, V. et al. (2020) An endophytic Fusarium-legume association is partially dependent on the common symbiotic signalling pathway. New Phytol. 226, 1429-1444

51. Treutter, D. (2006) Significance of flavonoids in plant resistance: a review. Environ. Chem. Lett. 4, 147-157

52. Chen, J. et al. (2019) Sclerotinia sclerotiorum circumvents flavonoid defenses by catabolizing flavonol glycosides and aglycones. Plant Physiol. 180, 1975-1987

53. Dakora, F.D. et al. (1993) Alfalfa (Medicago sativa L.) root exudates contain isoflavonoids in the presence of Rhizobium meliloti. Plant Physiol. 101, 819-824

54. Pankhurst, C.E. and Biggs, D.R. (1980) Sensitivity of rhizobium to selected isoflavonoids. Can. J. Microbiol. 26, 542-545

55. Stringlis, I.A. et al. (2019) The age of coumarins in plantmicrobe interactions. Plant Cell Physiol. 60, 1405-1419

56. Stringlis, I.A. et al. (2018) MYB72-dependent coumarin exudation shapes root microbiome assembly to promote plant health. Proc. Natl. Acad. Sci. U. S. A. 115, E5213-E5222

57. Voges, M.J.E.E.E. et al. (2019) Plant-derived coumarins shape the composition of an arabidopsis synthetic root microbiome. Proc. Natl. Acad. Sci. U. S. A. 116, 12558-12565

58. Kai, K. et al. (2008) Scopoletin is biosynthesized via orthohydroxylation of feruloyl CoA by a 2-oxoglutarate-dependent dioxygenase in Arabidopsis thaliana. Plant J. 55, 989-999

59. Vanholme, R. et al. (2019) COSY catalyses trans-cis isomerization and lactonization in the biosynthesis of coumarins. Nat. Plants 5, 1066-1075

60. Siwinska, J. et al. (2018) Scopoletin 8-hydroxylase: a nove enzyme involved in coumarin biosynthesis and iron-deficiency responses in arabidopsis. J. Exp. Bot. 69, 1735-1748

61. Tsai, H.-H. et al. (2018) Scopoletin 8-hydroxylase-mediated fraxetin production is crucial for iron mobilization. Plant Physiol. 177, 194-207
62. Rajniak, J. et al. (2018) Biosynthesis of redox-active metaboites in response to iron deficiency in plants. Nat. Chem. Biol. $14,442-450$

63. Le Roy, J. et al. (2016) Glycosylation is a major regulator of phenylpropanoid availability and biological activity in plants. Front. Plant Sci. 7, 1-19

64. Werner, C. and Matile, P. (1985) Accumulation of coumarylglucosides in vacuoles of barley mesophyll protoplasts. J. Plant Physiol. 118, 237-249

65. Taguchi, G. et al. (2000) Scopoletin uptake from culture medium and accumulation in the vacuoles after conversion to scopolin in 2,4-D-treated tobacco cells. Plant Sci. 151, 153-161

66. Kai, K. et al. (2006) Accumulation of coumarins in Arabidopsis thaliana. Phytochemistry 67, 379-386

67. Ziegler, J. et al. (2017) Arabidopsis transporter ABCG37/PDR9 contributes primarily highly oxygenated coumarins to root exudation. Sci. Rep. 7, 1-11

68. Ahn, Y.O. et al. (2010) Scopolin-hydrolyzing $\beta$-glucosidases in roots of arabidopsis. Plant Cell Physiol. 51, 132-143

69. Kobayashi, T. and Nishizawa, N.K. (2012) Iron uptake, translocation, and regulation in higher plants. Annu. Rev. Plant Biol. 63, 131-152

70. Römheld, $V$ and Marschner, $H$. (1986) Evidence for a specific uptake system for iron phytosiderophores in roots of grasses. Plant Physiol. 80, 175-180

71. Santi, S. and Schmidt, W. (2009) Dissecting iron deficiencyinduced proton extrusion in arabidopsis roots. New Phytol. 183, 1072-1084

72. Eide, D. et al. (1996) A novel iron-regulated metal transporter from plants identified by functional expression in yeast. Proc. Natl. Acad. Sci. U. S. A. 93, 5624-5628

73. Robinson, N.J. et al. (1999) A ferric-chelate reductase for iron uptake from soils. Nature 397, 694-697

74. Schwarz, B. and Bauer, P. (2020) FIT, a regulatory hub for iron deficiency and stress signaling in roots, and FIT-dependent and -independent gene signatures. J. Exp. Bot. 71, 1694-1705

75. Lan, P. et al. (2011) iTRAQ protein profile analysis of arabidopsis roots reveals new aspects critical for iron homeostasis. Plant Physiol. 155, 821-834

76. Zamioudis, C. et al. (2014) B-Glucosidase BGLU42 is a MYB72-dependent key regulator of rhizobacteria-induced systemic resistance and modulates iron deficiency responses in arabidopsis roots. New Phytol. 204, 368-379

77. Palmer, C.M. et al. (2013) MYB10 and MYB72 are required for growth under iron-limiting conditions. PLoS Genet. 9, e1003953

78. Fourcroy, P. et al. (2014) Involvement of the ABCG37 transporter in secretion of scopoletin and derivatives by arabidopsis roots in response to iron deficiency. New Phytol. 201, 155-167

79. Schmid, N.B. et al. (2014) Feruloyl-CoA 6'-hydroxylase1dependent coumarins mediate iron acquisition from alkaline substrates in arabidopsis. Plant Physiol. 164, 160-172

80. Fourcroy, P. et al. (2016) Facilitated Fe nutrition by phenolic compounds excreted by the arabidopsis ABCG37/PDR9 transporter requires the IRT1/FRO2 high-affinity root $\mathrm{Fe}^{2+}$ transport system. Mol. Plant 9, 485-488

81. Danhorn, T. and Fuqua, C. (2007) Biofilm formation by plantassociated bacteria. Annu. Rev. Microbiol. 61, 401-422

82. Reen, F.J. et al. (2018) Coumarin: a novel player in microbia quorum sensing and biofilm formation inhibition. Appl. Microbiol. Biotechnol. 102, 2063-2073

83. D'Almeida, R.E. et al. (2017) Comparison of seven structurally related coumarins on the inhibition of quorum sensing of Pseudomonas aeruginosa and Chromobacterium violaceum. Bioorg. Chem. 73, 37-42

84. Lee, J.H. et al. (2014) Coumarins reduce biofilm formation and the virulence of Escherichia coli 0157:H7. Phytomedicine 21, 1037-1042

85. Zeng, Z. et al. (2008) Virtual screening for novel quorum sensing inhibitors to eradicate biofilm formation of Pseudomonas aeruginosa. Appl. Microbiol. Biotechnol. 79, 119-126

86. Yang, L. et al. (2016) New insights into the antibacterial activity of hydroxycoumarins against Ralstonia solanacearum. Molecules 21, 1-13

87. Pruteanu, M. et al. (2020) Common plant flavonoids prevent the assembly of amyloid curli fibres and can interfere with 
bacterial biofilm formation. Environ. Microbiol. Published online August 31, 2020. https://doi.org/10.1111/1462-2920.15216

88. Sun, H. et al. (2014) Scopoletin is a phytoalexin against Alternaria alternata in wild tobacco dependent on jasmonate signalling. J. Exp. Bot. 65, 4305-4315

89. Beyer, S.F. et al. (2019) The arabidopsis non-host defenceassociated coumarin scopoletin protects soybean from Asian soybean rust. Plant J. 99, 397-413

90. Payá, M. et al. (1992) Interactions of a series of coumarins with reactive oxygen species. Biochem. Pharmacol. 44, 205-214

91. Michalet, S. et al. (2013) Phytochemical analysis of mature tree root exudates in situ and their role in shaping soil microbia communities in relation to tree $\mathrm{N}$-acquisition strategy. Plant Physiol. Biochem. 72, 169-177

92. Nasir, F. et al. (2019) Strigolactones shape the rhizomicrobiome in rice (Oryza sativa). Plant Sci. 286, 118-133

93. Liu, F. et al. (2020) Overexpression of strigolactone-associated genes exerts fine-tuning selection on soybean rhizosphere bacterial and fungal microbiome. Phytobiomes J. 4, 239-251

94. Scharf, B.E. et al. (2016) Chemotaxis signaling systems in model beneficial plant-bacteria associations. Plant Mol. Biol. 90, 549-559

95. Singh, A. et al. (2016) Rice seed priming with picomolar rutin enhances rhizospheric Bacillus subtilis CIM colonization and plant growth. PLOS ONE 11, 1-17

96. Caetano-Anollés, G. et al. (1988) Chemotaxis of Rhizobium meliloti to the plant flavone luteolin requires functional nodulation genes. J. Bacteriol. 170, 3164-3169

97. Segarra, G. et al. (2009) MYB72, a node of convergence in induced systemic resistance triggered by a fungal and a bacteria beneficial microbe. Plant Biol. 11, 90-96

98. Pieterse, C.M.J. et al. (2014) Induced systemic resistance by beneficial microbes. Annu. Rev. Phytopathol. 52, 347-375

99. Verhagen, B.W.M. et al. (2004) The transcriptome of rhizobacteria-induced systemic resistance in arabidopsis. Mol. Plant-Microbe Interact. 17, 895-908
100. Martinez-Medina, A. et al. (2016) Recognizing plant defense priming. Trends Plant Sci. 21, 818-822

101. Yang, W. et al. (2016) Rutin-mediated priming of plant resistance to three bacterial pathogens initiating the early SA signa pathway. PLOS ONE 11, 1-15

102. Zamioudis, C et al. (2015) Rhizobacterial volatiles and photosynthesis-related signals coordinate MYB72 expression in arabidopsis roots during onset of induced systemic resistance and iron-deficiency responses. Plant J. 84, 309-322

103. Van der Ent, S. et al. (2008) MYB72 is required in early signaling steps of rhizobacteria-induced systemic resistance in arabidopsis. Plant Physiol. 146, 1293-1304

104. Martínez-Medina, A. et al. (2017) Airborne signals from Trichoderma fungi stimulate iron uptake responses in roots resulting in priming of jasmonic acid-dependent defences in shoots of Arabidopsis thaliana and Solanum lycopersicum. Plant Cell Environ. 40, 2691-2705

105. Verbon, E.H. et al. (2019) Rhizobacteria-mediated activation of the $\mathrm{Fe}$ deficiency response in arabidopsis roots: impact on $\mathrm{Fe}$ status and signaling. Front. Plant Sci. 10, 1-12

106. Wang, C. et al. (2018) The art of self-control - autoregulation o plant-microbe symbioses. Front. Plant Sci. 9, 1-8

107. Tsikou, D. et al. (2018) Systemic control of legume susceptibility to rhizobial infection by a mobile microRNA. Science (80-. ) 236 , 233-236

108. Shine, M.B. et al. (2019) Glycerol-3-phosphate mediates rhizobia-induced systemic signaling in soybean. Nat. Commun 10, 5303

109. Knoblauch, M. et al. (2015) Multispectral phloem-mobile probes: properties and applications. Plant Physiol. 167, 1211-1220

110. Buer, C.S. et al. (2007) Flavonoids are differentially taken up and transported long distances in arabidopsis. Plant Physiol. $145,478-490$

111. Sasse, J. et al. (2015) Asymmetric localizations of the ABC transporter PaPDR1 trace paths of directional strigolactone transport. Curr. Biol. 25, 647-655 